\title{
Genotyping of Coxiella burnetii strains detected in cattle from a nationwide survey in Korea
}

\author{
Yo-Han Kim ${ }^{1,2}$, Doo Kim ${ }^{2, *}$ \\ ${ }^{I}$ Cooperative Department of Veterinary Medicine, Faculty of Agriculture, Iwate University, Morioka 020-8550, Japan \\ ${ }^{2}$ Laboratory of Veterinary Internal Medicine, College of Veterinary Medicine, Kangwon National University, Chuncheon 24341, Korea
}

\begin{abstract}
A total of 123 Coxiella burnetii strains detected in cattle from a nationwide survey in Korea were classified into five genomic groups: I ( $0.8 \%)$, II (14.6\%), III (12.2\%), IV (66.7\%), and V (5.7\%). Acute Q-fever strains (Groups I, II, and III) comprised 27.6\% and chronic strains (Groups IV and V) comprised $72.4 \%$. At a dairy farm where both types were present, the detection rate was 6.7-times higher than that of another farm where only chronic strains were detected. Both acute and chronic Q-fever strains are widespread in Korea, and their presence could represent an active transmission to livestock and humans.
\end{abstract}

Keywords: Coxiella burnetii; Korea; Cattle; Genomic groups

Coxiella burnetii is an obligate, intracellular, rickettsial organism and the causative agent of Query fever (Q-fever), a zoonosis that occurs worldwide. $C$. burnetii infection is generally asymptomatic in animals, and ruminants are considered to be the primary source of infection in humans [7]. However, the potential role of cattle as a source of zoonotic C. burnetii transmission is incompletely described in Korea. Therefore, elucidation of $C$. burnetii infection status in cattle is required to address the potential risk to public health in Korea.

Denison et al. [2] previously developed an algorithm flowchart for differentiation of $C$. burnetii isolates into one of five genomic groups, using a repetitive-element polymerase chain reaction (PCR)-based differentiation method. Among those, genomic Groups I, II, and III are associated with acute Q-fever human isolates, whereas genomic Groups IV and V are associated with chronic Q-fever human endocarditis isolates. The objective of the present study was to investigate the genomic grouping of $C$. burnetii strains detected from blood and milk samples on a nationwide scale in Korea by applying the repetitive-element PCR-genotyping method.

Samples were collected from August 2010 to October 2011. Eight hundred and seven blood samples of 622 native Korean cattle and 185 dairy cows were obtained along with 170 individual milk samples obtained from dairy cows, and 348 bulk tank milk (BTM) samples from dairy herds, for the detection of C. burnetii as reported previously [4]. C. burnetii strains were detected by real-time PCR assay using primers designed to amplify a $243 \mathrm{bp}$ fragment of the IS1111 transposon-like repetitive gene. Assay specificity was confirmed by subjecting the PCR products to agarose gel electrophoresis, DNA sequencing, and melting curve analysis as reported previously [4]. The primer IS1111-1 (5'-ACTGCGTTGGGATACCCATC-3') was designed to anneal to a conserved sequence present in all insertion sequence (IS) genes within the Nine Mile Phase I (9Mi/I) genome, and was paired with all four primers (IS5; 5'-ACTGCGTTGGGATACCCATC-3', IS9; 5'-GCCTCA GCCGATTTCGAG-3', IS14; 5'-TGCTACCAACAGAC TTACGGCA-3', and IS20; 5'-ACGTCAATTACATCGAG CATTCA-3'), which were selected to bind to each IS element [2]. C. burnetii strains were classified into the five genomic groups (I to $\mathrm{V}$ ) by following a previously reported classification algorithm [2]. For example, C. burnetii strains belonging to genomic Groups I, II, and III were classified as acute Q-fever strains, and those belonging to genomic Groups IV and V were classified as chronic Q-fever strains.

A total of 130 C. burnetii strains were detected from BTM $(\mathrm{n}=84)$, individual milk $(\mathrm{n}=27)$, and blood $(\mathrm{n}=19)$ samples, and excluding 7 unclassified strains from blood samples, 123 strains were differentiated by using repetitive-element PCR 
genotyping (Table 1, panel A in Fig. 1). Of the 123 C. burnetii strains, 1 (0.8\%), 18 (14.6\%), $15(12.2 \%), 82(66.7 \%)$, and 7 (5.7\%) were classified into genomic Groups I, II, III, IV, and V, respectively. Acute Q-fever strains comprised $27.6 \%$ of the differentiated total (34/123) and chronic Q-fever strains comprised $72.4 \%$ of the same (89/123). Regarding the individual milk samples, the genomic groups were summarized in detail for Farms No. 1 and No. 2 (panel B in Fig. 1). Farm No. $1(n=67)$ showed a relatively higher detection rate than that for Farm No. $2(\mathrm{n}=103)(32.8 \%$ vs. 4.9\%). Genomic Groups II and III (acute Q-fever) were detected only on Farm No. 1 (both $7.5 \%$ of the farm's total), whereas genomic Groups IV and V (chronic Q-fever) were detected on both Farm No. 1 and No. 2 (17.9\% and $4.9 \%$ of the farms' total, respectively).

Previously, Kim and Kim [4] reported, using a substantially large sample size $(\mathrm{n}=1,325)$, that $C$. burnetii infection in cattle is widespread in nine provinces of Korea. Specifically, the detection rates in their study showed a range of $0 \%$ to $12.5 \%$ in the blood samples, $4.9 \%$ to $32.8 \%$ in the individual milk

Table 1. Sample type and Korean province of collection of 123 genotyped Coxiella burnetii strains

\begin{tabular}{|c|c|c|}
\hline Type & No. & Province \\
\hline Bulk tank milk & 84 & $\begin{array}{l}\text { Chonnam, Chonbuk, Chungnam, } \\
\text { Chungbuk, Gangwon, Gyeonggi, } \\
\text { Gyeongnam, Gyeongbuk, Jeju }\end{array}$ \\
\hline Individual milk & 27 & Chungnam, Gyeonggi \\
\hline Blood & 12 & Chungnam, Gangwon, Gyeonggi, Jeju \\
\hline Total & 123 & \\
\hline
\end{tabular}

samples, and $10.0 \%$ to $38.0 \%$ in the BTM samples [4]. Furthermore, the detection rate of their BTM samples was lower than those reported for European countries (51.7-69.7\%) $[1,5,9]$ and the United States (93.2-94.3\%) [3]. Regardless, the prevalence of Q-fever is reported to be continuously increasing in dairy herds [3]; thus, periodic investigations are needed to monitor the prevalence of $C$. burnetii infection in Korean livestock.

The 20 unique copies of the IS1111 transposase in the complete genome sequence of $9 \mathrm{Mi} / \mathrm{I}$ have been identified [8]. C. burnetii isolates from acute and chronic disease patients can be differentiated into one of five genomic groups, based on the presence or absence of four different IS elements [2]. In addition, $C$. burnetii isolates associated with acute and chronic diseases have induced genogroup-specific virulence in an animal challenge model. For example, the acute Q-fever associated strains (Group I isolates) caused the most rapidly progressive disease in mice, whereas the chronic Q-fever associated strains (Groups IV and V isolates) caused a slower progression of disease in mice [6]. In this study, the detection rate of chronic Q-fever strains was higher than that of the acute Q-fever strains. Although we did not associate genomic groups with bacterial shedding or transmission among the cattle in our study, it is clear that chronic infection in cattle is widespread and that acute infection is present in Korea.

Regarding the individual milk samples obtained from Farms No. 1 and No. 2 (geographically distinct areas), there was a high contrast between their detection rates and genotypes. On Farm No. 2, only chronic Q-fever strains were detected; conversely, acute and chronic Q-fever strains were detected simultaneously on Farm No. 1, with detection rate 6.7 times higher than that in


Fig. 1. Clinical type and genomic group of Coxiella burnetii strains detected in Korean cattle. (A) Clinical types of $C$. burnetii strains in bulk tank milk (BTM), individual cow milk, and serum samples. 'Acute' denotes acute Q-fever strains belonging to genomic Groups I, II, and III, and 'chronic' denotes chronic Q-fever strains belonging to genomic Groups IV and V. The numbers within each column indicate the detection rate of each clinical type. (B) Genomic Groups of $C$. burnetii strains in the individual cow milk samples from two dairy herds (Farms No. 1 and No. 2). Genomic Group I was not detected at either farm. The numbers within each column indicate the detection rate of each genomic group. 
Farm No. 1. With regard to Farm No. 1, the detection of acute Q-fever strains may imply ongoing active bacterial transmission among the cattle in the farm's dairy herd. The results suggest that the implementation of more careful management strategies may be required for dairy herds in which acute infections are identified.

In conclusion, the present study undertook the genotyping of C. burnetii strains on a nationwide scale in Korea. Both acute and chronic Q-fever C. burnetii strains were detected in cattle, indicating the presence of a persistent transmission source of Q-fever in livestock and the potential for transmission to humans, through direct or indirect exposure to bacteria. Therefore, further studies are needed to elucidate $C$. burnetii transmission characteristics among livestock and humans and to investigate the pathogenic implications of the presence of acute and chronic forms of Q-fever.

\section{Acknowledgments}

This study was supported by a 2017 research grant from Kangwon National University (No. 520170373) and a grant (Z-AD13-2-10-11-03) from Animal and Plant Quarantine Agency, Republic of Korea.

\section{Conflict of Interest}

The authors declare no conflicts of interest.

\section{References}

1. Astobiza I, Ruiz-Fons F, Piñeno A, Barandika JF, Hurtado A, García-Pérez AL. Estimation of Coxiella burnetii prevalence in dairy cattle in intensive systems by serological and molecular analyses of bulk-tank milk samples. J Dairy Sci 2012, 95, 1632-1638.

2. Denison AM, Thompson HA, Massung RF. IS 1111 insertion sequences of Coxiella burnetii: characterization and use for repetitive element PCR-based differentiation of Coxiella burnetii isolates. BMC Microbiol 2007, 7, 91.

3. Kim SG, Kim EH, Lafferty CJ, Dubovi E. Coxiella burnetii in bulk tank milk samples, United States. Emerg Infect Dis 2005, 11, 619-621.

4. Kim YH, Kim D. Detection of Coxiella burnetii in cattle. J Vet Clin 2015, 32, 504-507.

5. Muskens J, van Engelen E, van Maanen C, Bartels C, Lam TJ. Prevalence of Coxiella burnetii infection in Dutch dairy herds based on testing bulk tank milk and individual samples by PCR and ELISA. Vet Rec 2011, 168, 79.

6. Russell-Lodrigue KE, Andoh M, Poels MW, Shive HR, Weeks BR, Zhang GQ, Tersteeg C, Masegi T, Hotta A, Yamaguchi T, Fukushi H, Hirai K, McMurray DN, Samuel JE. Coxiella burnetii isolates cause genogroup-specific virulence in mouse and guinea pig models of acute $\mathrm{Q}$ fever. Infect Immun 2009, 77, 5640-5650.

7. Schimmer B, Dijkstra F, Vellema P, Schneeberger PM, Hackert V, ter Schegget R, Wijkmans C, van Duynhoven Y, van der Hoek W. Sustained intensive transmission of Q fever in the south of the Netherlands, 2009. Euro Surveill 2009, 14, 19210.

8. Seshadri R, Paulsen IT, Eisen JA, Read TD, Nelson KE, Nelson WC, Ward NL, Tettelin H, Davidsen TM, Beanan MJ, Deboy RT, Daugherty SC, Brinkac LM, Madupu R, Dodson RJ, Khouri HM, Lee KH, Carty HA, Scanlan D, Heinzen RA, Thompson HA, Samuel JE, Fraser CM, Heidelberg JF. Complete genome sequence of the Q-fever pathogen Coxiella burnetii. Proc Natl Acad Sci U S A 2003, 100, 5455-5460.

9. Valergakis GE, Russell C, Grogono-Thomas R, Bradley AJ, Eisler MC. Coxiella burnetii in bulk tank milk of dairy cattle in south-west England. Vet Rec 2012, 171, 156. 